\title{
A two-dimensional molecular dynamics simulation of thin film growth by oblique deposition
}

\author{
Liang Dong, Richard W. Smith, ${ }^{\text {a) }}$ and David J. Srolovitz ${ }^{\text {b) }}$ \\ Department of Materials Science and Engineering, University of Michigan, Ann Arbor, \\ Michigan 48109-2136
}

(Received 20 June 1996; accepted for publication 8 August 1996)

\begin{abstract}
Atomistic, molecular dynamics simulations are employed to investigate the relationship between film microstructure and deposition conditions (substrate temperature, deposition kinetic energy, and deposition angle). Increasing substrate temperature and deposition kinetic energy leads to fewer voids, smaller voids, smoother surfaces, and higher film density. As the deposition angle increases, the film microstructure changes from a dense film, with few voids, to a microstructure in which nearly colinear tracks of elongated voids form and, finally, to a highly porous structure of well-formed columns. The angle along which the voids are elongated and the orientation of the void tracks are the same and increase monotonically with the deposition angle (the column angles follow the same trend as the deposition angle). Void formation, void alignment into tracks, and the columnar structure are all attributable to shadowing effects, which become more pronounced with increasing deposition angle. The variation of the column/void track angle $\beta$ with deposition angle $\alpha$ fits well with the classical tangent law at low angles, but is overpredicted by the tangent law at $\alpha>60^{\circ}$, consistent with experiment. The column angle $\beta$ decreases slowly with increasing deposition kinetic energy due to increased surface mobility. (C) 1996 American Institute of Physics. [S0021-8979(96)01922-6]
\end{abstract}

\section{INTRODUCTION}

In physical vapor deposition (PVD), atoms are deposited from a gas phase onto a substrate without the decomposition of one chemical species into other species, as in chemical vapor deposition. The parameters that describe the PVD deposition process include deposition rate, deposition angle (i.e., the angle the velocity vector of a depositing atom makes with the substrate normal), the distribution of deposition angles (i.e., the degree of collimation of the deposition beam), the kinetic energy of the depositing atoms, the substrate temperature, etc. These deposition parameters all play an important role in determining the microstructure and physical properties of PVD-grown films. The effects of each of these parameters have received considerable experimental attention. For example, the microstructure of PVD grown films has been divided into three distinct zones, based upon the substrate temperature during deposition. ${ }^{1}$ At substrate temperatures below approximately $0.3 T_{m}\left(T_{m}\right.$ is the melting point), the films tend to exhibit a very porous columnar microstructure. This porous structure is increasingly obvious as the deposition angle becomes larger (i.e., increasingly oblique). Experimental observations suggest that the angle of orientation of these columns (relative to the substrate normal) $\beta$, in these so-called zone I microstructures, is generally smaller than the deposition angle (relative to the substrate normal) $\alpha$ : i.e., the columnar structure is oriented more nearly perpendicular to the substrate than the deposition flux. Nieuwenhuizen and Haanstra ${ }^{2}$ were able to describe the re-

${ }^{a}$ Current address: 2403 Corteland Drive, Pittsburgh, Pennsylvania 15241.

${ }^{b)}$ Electronic mail: srol@umich.edu lationship between the column orientation and the deposition angle in their experimental results using the following empirical relation:

$\tan \alpha=2 \tan \beta$.

Equation (1) is widely known as the "tangent law." Experimentally, this relationship is found to be valid for deposition angles $\alpha \leqslant 60^{\circ}{ }^{3,4}$ Additional experimental studies ${ }^{3,5}$ indicate that decreasing deposition angles and/or increasing substrate temperature result in an increase in film density. In the present paper, we employ atomistic, molecular dynamics simulations to investigate the role of several PVD deposition parameters (deposition angle, kinetic energy, and substrate temperature) on the microstructure (density, roughness, and column orientation) of growing films.

Many atomistic simulations studies of film growth have been performed to date. ${ }^{6-12}$ These simulations have been based upon Monte Carlo (MC), molecular dynamics (MD) and ballistic deposition models. Paik et al. ${ }^{6}$ performed a two-dimensional MD simulation to study the formation of columnar microstructures in thin films grown by PVD. These authors investigated the effects of substrate temperature, beam energy, and substrate roughness on the growth of columnar microstructures. They found that the columnar microstructure grew out of the surface roughness and, under their deposition conditions, no columnar microstructure was observed when the substrate was flat. The columnar microstructures that they observed at low substrate temperatures were well described by the tangent law for deposition angles in the range $30^{\circ} \leqslant \alpha \leqslant 60^{\circ}$. They also observed that film density decreased with increasing deposition angle.

Based on geometrical arguments, Tait et al., ${ }^{7}$ derived another expression relating the column angle to the deposition angle for the case of limited surface diffusion: 


$$
\beta=\alpha-\sin ^{-1}\left[\frac{1-\cos \alpha}{2}\right] .
$$

This expression was shown to yield a better fit to the experimental data at high deposition angles than the tangent law. They used a ballistic deposition model to verify their expressions. Müller ${ }^{8}$ employed a two-dimensional Monte Carlo simulation to investigate the effects of deposition rate and substrate temperature on oblique deposition. His results show that increasing temperature leads to a continuous transition from a porous film with a columnar microstructure to a densely packed film. The transition temperature was shown to increase with deposition rate.

In a recent paper, Smith and Srolovitz ${ }^{13}$ reported the results of a series of two-dimensional MD simulations examining the effects of substrate temperature and kinetic energy of the depositing species on void formation during the PVD growth of thin films. This study was limited to the special case in which the deposition flux was oriented perpendicular to the substrate. This study showed how void formation was a direct result of the roughening process and shadowing effects.

In the present paper, we employ the molecular dynamics simulation method to investigate the effects of deposition conditions on the microstructure of a growing film. In particular, we focus on understanding the relationship between roughening and porosity development during film growth and how deposition angle effects these two important microstructural parameters. In the next section, we outline the simulation method employed. We then investigate the effects of temperature and deposition kinetic energy on film growth at fixed deposition angle, since these deposition parameters are known to have a pronounced effect on the roughness and porosity of the growing film. Next, we examine the role of deposition angle on the roughness and porosity of the film. We also use this data to evaluate the validity of the different predictions for the evolution of column angle with deposition angle. We find that the column angle depends on the size of the system at large deposition angle. The simulation results demonstrate that the column angle depends on deposition energy in a manner not described by any of the theoretical or empirical models. We show that this effect is associated with the role that deposition energy plays in modifying the roughness of the growth surface and thereby the degree of shadowing that occurs.

\section{METHOD}

The molecular dynamics simulation method employed for the present film growth simulations is essentially identical to that used in a previous study. ${ }^{13}$ Therefore, we present only a brief outline of the method here. The MD simulations were performed by integrating Newton's classical equation of motion for each atom (in two dimensions) forward in time. The potential energy of the system was expressed as a sum over all pairs of atoms using the classical Lennard-Jones pair potential,

$$
U\left(r_{i j}\right)=\epsilon\left[\left(\frac{r_{0}}{r_{i j}}\right)^{12}-2\left(\frac{r_{0}}{r_{i j}}\right)^{6}\right],
$$

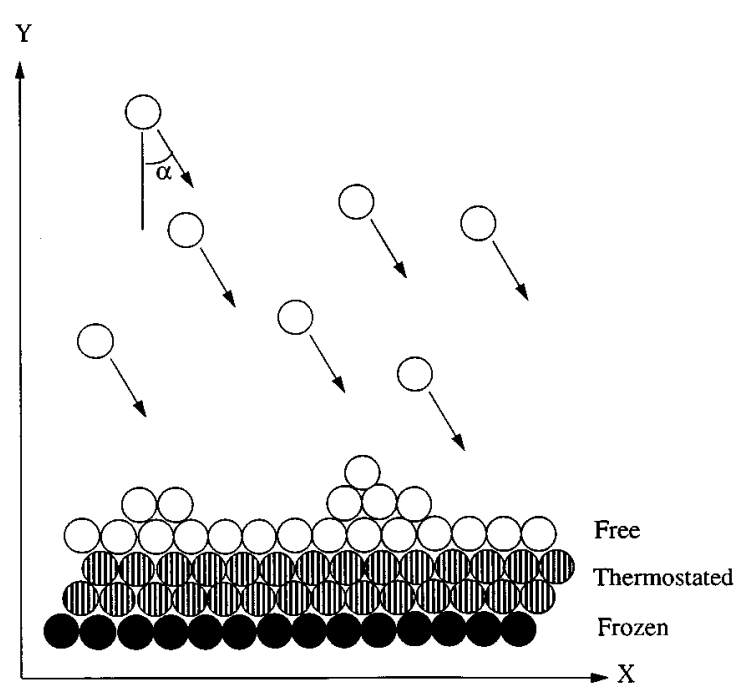

FIG. 1. Computational cell depicting the substrate, growing film and atoms impinging onto the surface. The bottom layer of the substrate is frozen, the two layers above it are thermostated and the atoms in subsequent layers are unconstrained. The thickness of the thermostated region increases with film thickness.

where $U\left(r_{i j}\right)$ is the interaction energy between atoms $i$ and $j$ separated by distance $r_{i j}$, and $\epsilon$ scales the strength of the interaction and $r_{0}$ is a characteristic length of the potential. This potential is smoothly cut off at $r=2.1 r_{0}$ and, except as noted, the substrate was $40 a_{0}$ wide, where $a_{0}$ is the nearest neighbor spacing. In this study, we focus on several generic features of film growth, rather than the properties of any particularly material. Therefore, we employ the simple Lennard-Jones potential, since it has been extensively characterized over many years.

In the present simulations, the physical parameters $\left(r_{0}, \epsilon\right.$ and $M$ ) were all set to unity. The velocity of the deposited atoms is $V=\sqrt{2 E_{b} / M}$ and the basic unit of time $\tau$ $=\sqrt{M r_{0}^{2} / \epsilon}$. The time step used in the integration of Newton's equation of motion was variable and was determined in terms of the maximum velocity at each time step $\Delta t=r_{0} /\left(200 V_{\text {max }}\right)$. Incident atoms are deposited at a rate of one per $0.75 \mathrm{MD}$ time units $(\tau)$. In order to give a physical feel for these parameters in terms of a real material, we have estimated these values for Ni. For Ni we find: $r_{0}=0.249 \mathrm{~nm}$, $\epsilon=0.74 \mathrm{eV}, M=9.7 \times 10^{-26} \mathrm{~kg}$, and $\tau=1.6 \times 10^{-13} \mathrm{~s}$. This yields a surface energy of order $2 \mathrm{~J} / \mathrm{m}^{2}$. While all of the results reported below are in terms of the fundamental parameters $r_{0}, \epsilon, M$, and $E_{b}$, the data can be converted to more physically meaningful numbers using the values for $\mathrm{Ni}$ quoted here.

The computational cell is two-dimensional and lies entirely within the $X Y$-plane. The $X$ direction is parallel to the substrate surface. Periodic boundary conditions are employed along this direction. The $Y$ direction is perpendicular to the substrate surface and open (or free) boundary conditions are employed on the $+Y$ surface. The atoms in the incident beam are all deposited onto the substrate at a predetermined angle with respect to the negative $Y$ direction (see Fig. 1). Substrate atoms are initially arranged on a triangular 
lattice and the substrate consists of four atomic layers. The atoms in the bottom layer of the substrate are frozen in space to prevent the substrate from being distorted or dissociated by the incident atoms and to prevent the entire system from translating through space due to the momentum absorbed from the deposition flux. Atoms on the next two layers of the substrate are "thermostated" in order to maintain the substrate at the desired temperature. As the film grows, it is necessary to increase the height of the thermostated region to prevent excessive heating of the film due to the kinetic and bond energy associated with incorporation of vapor atoms into the solid. The top layer of the substrate consists of "free" atoms, whose movements are completely determined by the atomic interactions. If the atoms in the deposition flux have sufficiently large kinetic energy, they may reflect off of the surface or eject other surface atoms back up into the vapor. These reflected or ejected atoms can interact with depositing atoms and destroy the collimated and monoenergetic nature of the flux (leading to atomic clustering in the vapor). This becomes increasingly likely as the kinetic energy of the depositing atoms increases or the binding energy of an atom to the surface decreases (e.g., large roughness or high temperature). This phenomenon occurs much less frequently in real film deposition since experimental deposition rates are typically very much smaller than those used in the present simulations, (which are rather high in order to minimize computational demands). In order to prevent this interaction from occurring, the atoms in the vapor above the film are searched for atoms with velocities differing from that at which the atoms are initially introduced. All such atoms are removed from the vapor.

One of the deficiencies of modeling thin film deposition using molecular dynamics is the limited time scale that can be studied. The fundamental time step is typically of order one hundredth of the vibrational period of the atoms in the solid. In order to overcome this problem, we employ several techniques: high deposition rates, relatively thin films, collimation of the flux, etc. We prevent gas phase reactions from occurring by (1) insuring that the depositing atoms do not interact with each other prior to reaching the substrate and (2) by removing atoms that are either ejected or reflected from the surface. While high deposition rates do not allow sufficient time for realistic atomic diffusion at typical deposition temperatures, this can be overcome, in part, by performing the simulations at somewhat elevated temperatures: note that there is an exponential increase in the surface diffusion length with increasing temperature and only an inverse square root variation with deposition rate. Temperature control is a problem in the present simulations for two reasons: (1) the deposition rate is fast and (2) thermal diffusion is slow. Slow thermal diffusion compared to that in the deposition of metals because heat can only be removed by phonons, while thermal diffusion is primarily controlled by electronic effects in metals. In order to prevent excessive heating during the deposition simulations, we thermostat the film near the deposition surface. This, coupled with the freezing of the atoms below the thermostated region, makes the simulation faster but does not unduly restrain bulk diffusion, which should be much slower than surface diffusion.
Additional simulations, where the atoms are thermostated all the way through the film yield nearly indistinguishable microstructures, consistent with the accepted notion that microstructure development during deposition is primarily controlled by transport in the near surface region.

Once the simulation is initialized, particle positions are recorded as a function of time and used to make MD "movies" of the growing films. The surface roughness of the film $R$ is also measured at several times during the deposition. Several definitions of surface roughness have been suggested in the literature. In the present study, we define $R=\left(L-L_{0}\right) / L_{0}$ where $L$ is the total length of the actual surface and $L_{0}$ is the width of the film in the $X$ direction. A perfectly flat surface, therefore, corresponds to $R=0$. The surface length is obtained by counting the number of atoms on the surface. In this paper, the calculations of surface roughness $R$ are based upon measurements made at five equally spaced time increments during the deposition of three independent simulations. The film roughness in the $\alpha=60^{\circ}$ and $75^{\circ}$ deposition simulations yielded voids of heights which were appreciable fractions of the final film thickness and, hence, this approach could not be employed. In these two cases, the surface roughness was measured only at the end of the simulations. No apparent dependence of the surface roughness on film thickness was observed for $\alpha<60^{\circ}$, except at early times. The density of the films was also measured at the end of each simulation. The normalized film density is defined as the ratio of the number of atoms contained in the film to the number of atoms contained in a perfect crystal of the same thickness as the film.

\section{RESULTS}

Several thin films grown at substrate temperatures of $T=0,0.050,0.075,0.100,0.150,0.200$, and $0.250 \epsilon / k_{B}$ (the bulk melting temperature for two dimensional Lennard-Jones is approximately $T_{m}=0.415 \epsilon / k_{B}{ }^{14,15}$ ) with a deposition kinetic energy of $E_{b}=0.80 \mathrm{\epsilon} /$ atom and a deposition angle of $45^{\circ}$ are shown in Fig. 2. A pronounced trend of decreasing void number and void size and increasing film density with increasing temperature is readily apparent. At low substrate temperatures $\left(T \leqslant 0.15 \epsilon / k_{B}\right)$, the voids are observed to be aligned in tracks which are tilted with respect to the film normal. Such tracks are less apparent at higher temperatures where the void density is low. The mechanism by which the void tracks form was discussed at length in Ref. 13, where it was shown to be a result of atomic shadowing and a pinchoff mechanism. Examination of Fig. 2 also shows that the roughness of the surface decreases with increasing temperature. These qualitative observations are made more quantitative in Fig. 3 which shows the variation of the film density and surface roughness with substrate temperature (each data point represents an average over three independent simulation runs). The density increases monotonically with increasing substrate temperature; in good agreement with experimental data and earlier simulations. ${ }^{5,6,8}$ The mean surface roughness decays monotonically with increasing substrate temperature. Both the increase of the film density and decrease of the surface roughness with increasing temperature at this oblique deposition angle is qualitatively very similar 


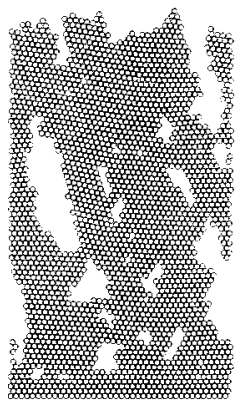

(c) $\mathrm{T}=0.075 \mathrm{\varepsilon} / \mathrm{k}$

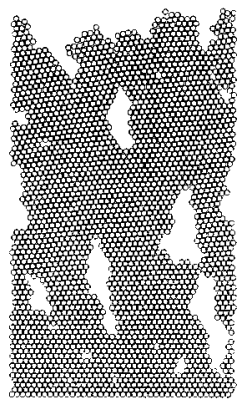

(e) $\mathrm{T}=0.15 \varepsilon / \mathrm{k}$

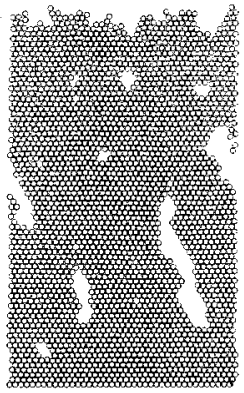

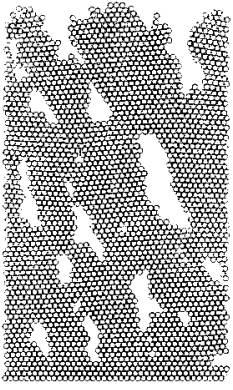

(d) $\mathrm{T}=0.10 \mathrm{~g} / \mathrm{k}$

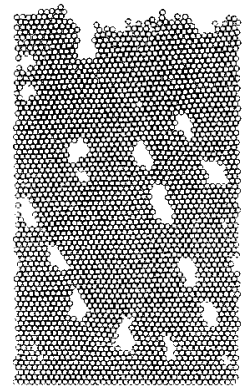

(f) $\mathrm{T}=0.20 \mathrm{~g} / \mathrm{k}$

(g) $\mathrm{T}=0.25 \mathrm{E} / \mathrm{k}$
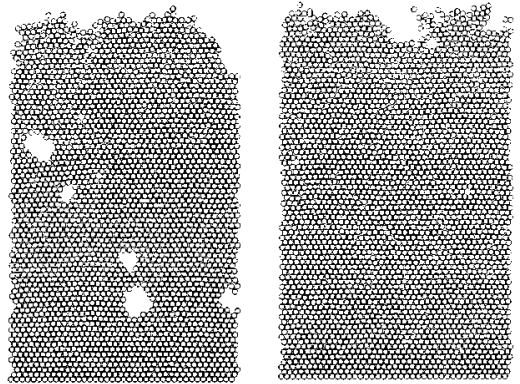

FIG. 2. Typical microstructures of films grown at various substrate temperatures with fixed deposition rate, $E_{b}=0.80 \epsilon$ and $\alpha=45^{\circ}$.

to that observed in film growth simulations where the deposition was at normal incidence with respect to the substrate. ${ }^{13}$

Microstructures of films grown at deposition kinetic energies of $E_{b}=0.10,0.45,0.80,1.15,1.50$, and $3.00 \mathrm{\epsilon}$ /atom with a constant substrate temperature of $T=0.125 \epsilon / k_{B}$ and a constant deposition angle of $\alpha=45^{\circ}$ are shown in Fig. 4. As the deposition kinetic energy increases, the number of voids, void size and surface roughness tend to decrease while the film density tends to increase. The dependence of the film density and surface roughness on deposition kinetic energy are shown in Fig. 5(a) and 5(b). The film density is observed to increase from approximately $85 \%$ at a deposition kinetic energy of $0.1 \mathrm{\epsilon}$ /atom to $97 \%$ at $3.0 \mathrm{\epsilon} / \mathrm{atom}$. The surface roughness decreases from approximately 1.48 at $E_{B}=0.1$ $\epsilon$ /atom to approximately 0.60 at $E_{B}=3.0 \mathrm{\epsilon}$ /atom. Comparison of Figs. 4 and 5 with Figs. 2 and 3 shows a striking resemblance between the effects of temperature and deposi- (a)

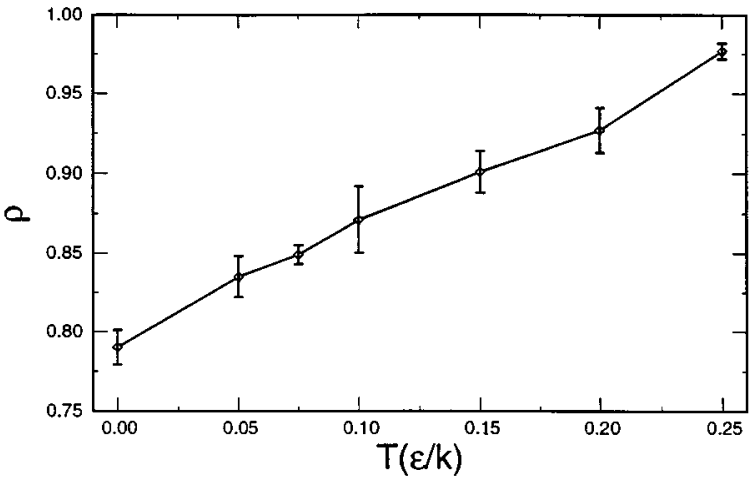

(b)

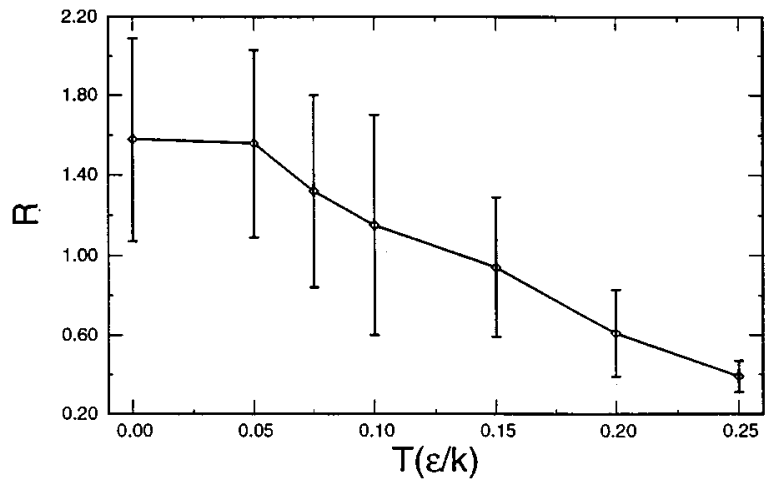

FIG. 3. Substrate temperature dependence of (a) the film density $\rho$ and (b) the mean surface roughness $R$, for films deposited at $E_{b}=0.80 \epsilon$ and $\alpha=45^{\circ}$. The error bars represent plus and minus one standard deviation of the data from (a) three independent simulations or from (b) five measurements per simulation (spaced equally in time) for three independent simulations.

tion kinetic energy on the film microstructures. This similarity between the variation in microstructure with temperature and deposition kinetic energy suggests that these two physical parameters play a similar role in the deposition process: namely, changing the atomic mobility on the growing surface. This observation is consistent with the observation made in the normal incidence film growth simulation. ${ }^{13}$

As described above, voids tend to be aligned into tracks at low temperature and low deposition kinetic energy. These tracks seem to be closely related with the column angle $\beta$, discussed in Sec. I. This can be seen clearly in Figs. 2(a) and (b) and Fig. 4(b). The variation of the void track angle $\beta$ appears to be negligible, but was difficult to determine with any reliability. However, the variation of the void track angle with deposition kinetic energy is measurable (as shown in Fig. 6). The track angles tend to decrease with increasing kinetic energy. There is no explicit dependence of the tangent law [Eq. (1)] or Eq. (2) on deposition kinetic energy. According to the tangent law $\beta=26.6^{\circ}$, and Eq. (2) predicts that $\beta=36.6^{\circ}$ when $\alpha=45^{\circ}$. Although we find some variation of $\beta$ with $E_{B}$, this variation is relatively small ( $7^{\circ}$ as compared with an average angle of approximately $25.9^{\circ}$ ) and the entire range of data is very close to the tangent law prediction and in poor agreement with the prediction of Eq. (2). 


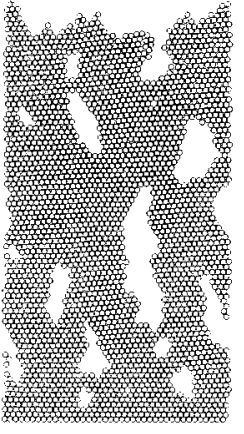

(d) $\mathrm{E}_{\mathrm{b}}=1.15 \mathrm{E}$

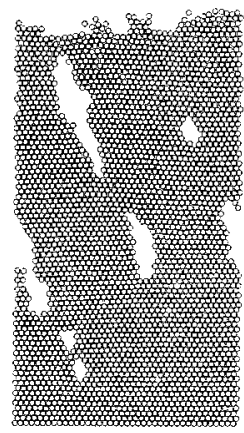

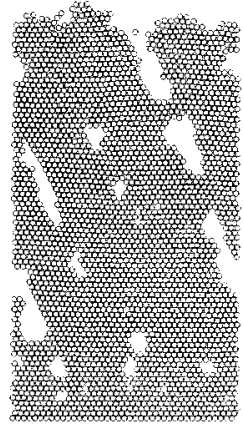

(e) $\mathrm{E}_{\mathrm{b}}=1.50 \varepsilon$

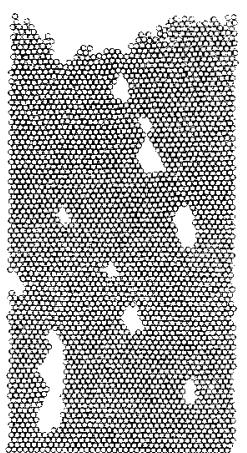

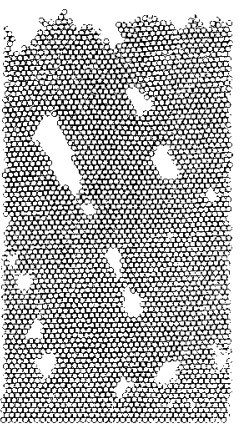

(f) $E_{b}=3.00 \varepsilon$

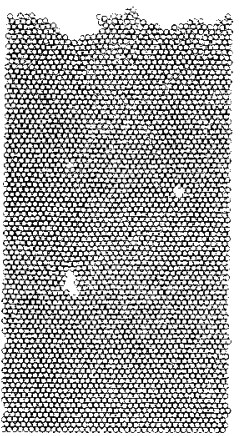

FIG. 4. Typical microstructures for films grown at various deposition kinetic energies onto a substrate at $T=0.125 \mathrm{\epsilon} / \mathrm{k}$ and $\alpha=45^{\circ}$.

Microstructures of films grown at deposition angles of $\alpha=0^{\circ}, 30^{\circ}, 37.5^{\circ}, 45^{\circ}, 60^{\circ}$, and $75^{\circ}$ with a deposition kinetic energy of $E_{b}=0.80 \mathrm{\epsilon}$ /atom and substrate temperature of $T=0.125 \epsilon / k_{B}$ are shown in Fig. 7 for substrates of width equal to $40 a_{0}$, except for the $\alpha=60^{\circ}$ and $75^{\circ}$ cases where substrates of width $120 a_{0}$ were used (see below). For normal incidence deposition $\left(\alpha=0^{\circ}\right)$, the film contains only a few, very small voids and/or vacancies. As the deposition angle increases, larger voids form and the density of the film drops monotonically. By $\alpha=45^{\circ}$, the voids begin to elongate and/or form into well defined tracks. A structure consisting of independent columns begins to form at $\alpha=60^{\circ}$ : however, this definition is not unique since the angle at which this transition to columns begins depends on the film thickness. The dependencies of film density and surface roughness on the deposition angle are shown in Fig. 8. When the deposition angle is less than $45^{\circ}$, the film density and surface roughness vary very slowly with the deposition angle. However, when the deposition angle exceeds $45^{\circ}$, the film density (roughness) begins to decrease (increase) rapidly with the deposition angle.

\section{DISCUSSION}

The general features of the variation of the film density and surface roughness with substrate temperature and deposition kinetic energy observed in the present oblique angle film deposition study are essentially the same as those reported in an earlier study ${ }^{13}$ where deposition occurred at nor- (a)

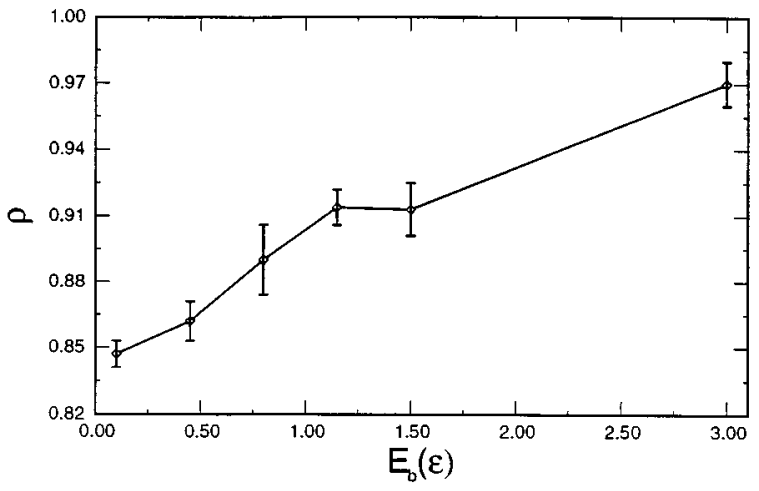

(b)

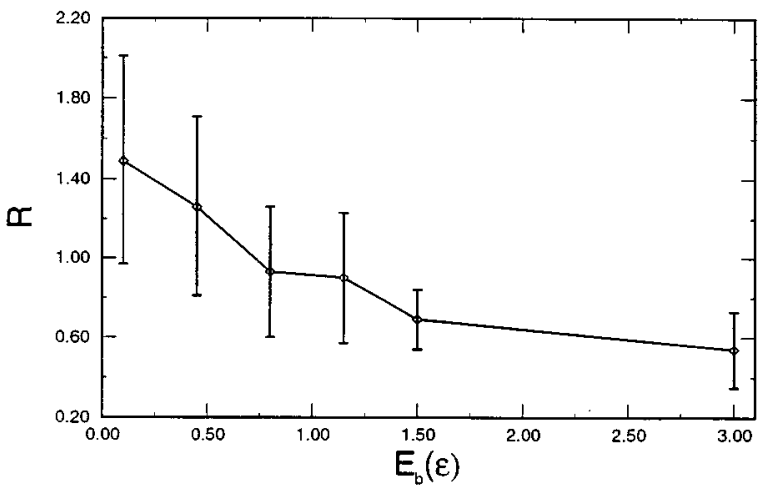

FIG. 5. Deposition kinetic energy dependence of (a) the film density $\rho$ and (b) the mean surface roughness $R$, for films deposited at $T=0.125 \epsilon / k$ and $\alpha=45^{\circ}$. The error bars represent plus and minus one standard deviation of the data from (a) three independent simulations or from (b) five measurements per simulation (spaced equally in time) for three independent simulations.

mal incidence $\left(\alpha=0^{\circ}\right)$. At all deposition angles, increasing the temperature or the deposition kinetic energy increases the mobility of the atoms at the surface. At normal incidence and at the deposition flux employed, increasing the surface atom mobility in this way is capable of overcoming the atomic shadowing effect that produces voids. As the angle of incidence $\alpha$ increases, shadowing is more effective and, hence,

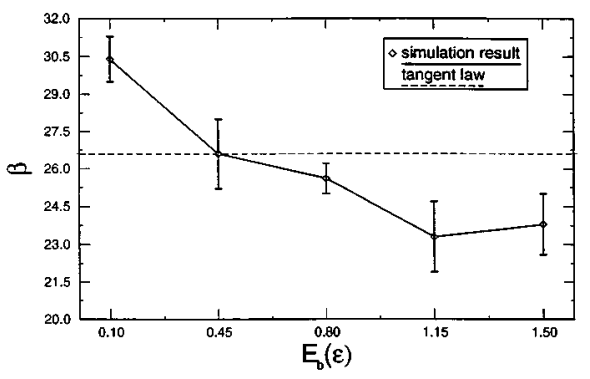

FIG. 6. Deposition kinetic energy dependence of the void track angle for films deposited at $T=0.125 \epsilon / k$ and $\alpha=45^{\circ}$. Error bars reflect the standard deviation of the measurements for three independent simulations. The dashed line represents the value of the void track angle predicted by the tangent law. 
(a) $\alpha=0^{\circ}$

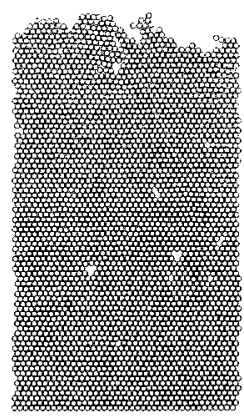

(c) $\alpha=37.5^{\circ}$

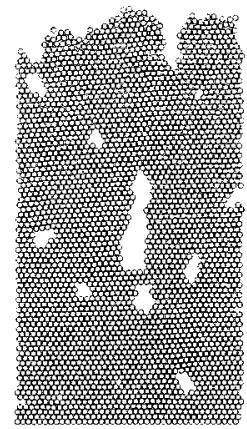

(b) $\alpha=30^{\circ}$

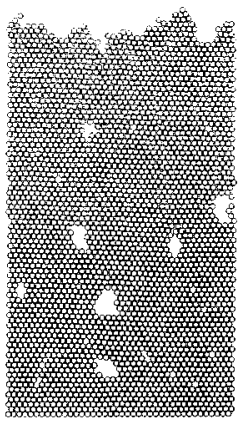

(d) $\alpha=45^{\circ}$

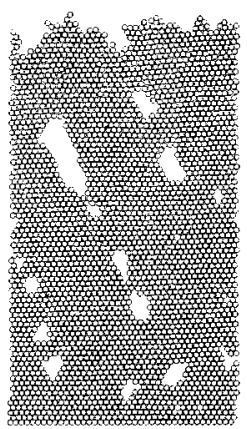

(e) $\alpha=60^{\circ}$

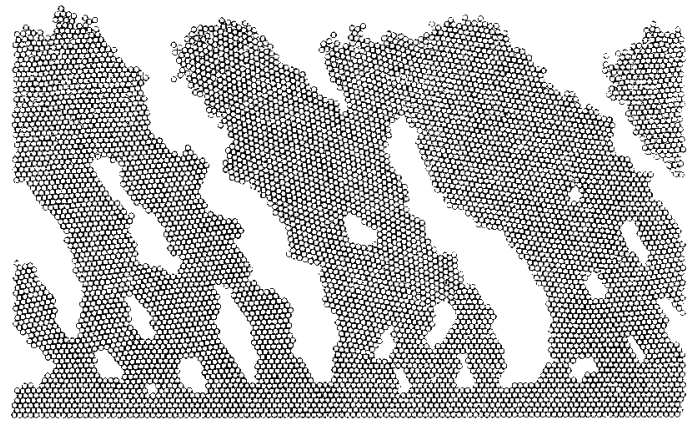

(f) $\alpha=75^{\circ}$

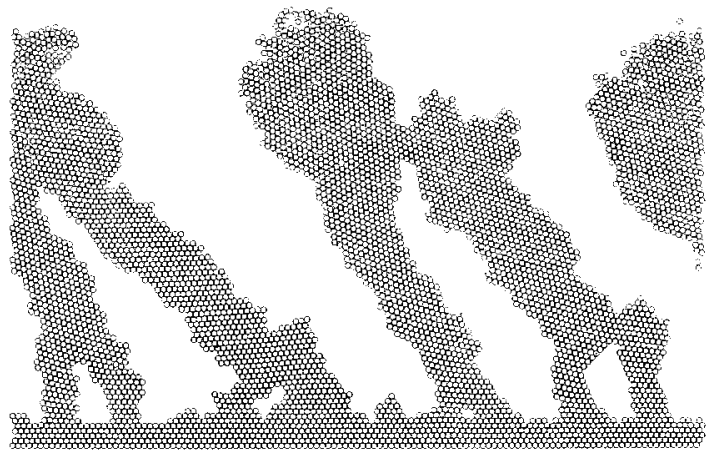

FIG. 7. Typical microstructures for films grown at various deposition angles onto a substrate at temperature $T=0.125 \epsilon / k$ and $E_{b}=0.80 \epsilon$.

the temperature and/or deposition kinetic energy must be further increased in order to produce a film with the same density as one produced at $\alpha=0^{\circ}$.

The formation of voids and void tracks is intimately related to the development of surface roughness, as described in mechanistic detail for normal incidence deposition in Ref. 13. Atoms which are being deposited in the vicinity of a surface depression will be attracted to the sides of the surface depression due to the normal atomic interactions (the Lennard-Jones potential here). As a result of these interactions, the sides of the surface depressions develop bumps, which shadow regions of the surface depression below. Eventually, these bumps grow into bridges over the surface depression; effectively cutting off the underlying atoms from any further flux and thereby pinching off voids (see Fig. 7 in Ref. 13 for a schematic illustration). If these bridges form below the top edge of the surface depressions (as is common), then a surface depression remains and a new void can form above the one that just pinched off at an angle consistent with the angle the elongated surface depression makes with the substrate normal. This aligns voids into void tracks and explains why the orientation of the elongated voids is the same as the orientation of the void tracks. The higher surface atom mobility associated with elevated temperature or deposition beam kinetic energy does two things: First, it prevents the formation of surface depressions in the first place due to diffusive smoothing ${ }^{16}$ and, second, it smoothes out the bumps on the edges of the surface depression before they form bridges by curvature driven surface diffusion. These effects are both borne out by the MD movies made of the deposition process. The difference between the normal and oblique deposition cases is that shadowing is much stronger at oblique deposition angles. If we consider a surface depression with sharp, vertical walls and turn off all atomic interactions, more atoms will impinge on the walls, and fewer make it all the way down to the bottom of the surface depression under oblique deposition angles than at normal incidence. This is a result of the fact that the angle of the surface depressions is not equal to the deposition angle, except at normal incidence (as predicted by the tangent rule). This produces more shadowing, less effective filling of the surface depression and, hence, more voids at oblique incidence than at normal incidence. Figure 9 compares the microstructures of the films grown at incidence angles of $\alpha=0^{\circ}$ and $\alpha=45^{\circ}$. Clearly, the film grown at $T=0 \epsilon / k_{B}$ [Fig. 9(a)] with $\alpha=45^{\circ}$ has a rougher surface, more voids, larger voids and tilted voids as compared with that grown at normal incidence. These same effects are observed at higher temperatures $\left[T=0.15 \epsilon / k_{B}\right.$ Fig. 9(b)], but with fewer and smaller voids overall, due to the enhanced surface atom diffusivity.

Before beginning our investigation of the effects of deposition angle on the film microstructure, we first investigated the effect of substrate width on the microstructure. The rationale for investigating this effect was that as $\alpha$ increases, the columns become increasingly tilted (i.e., $\beta$ increases) and, hence, at any $\alpha$ there will be some thickness at which 
(a)

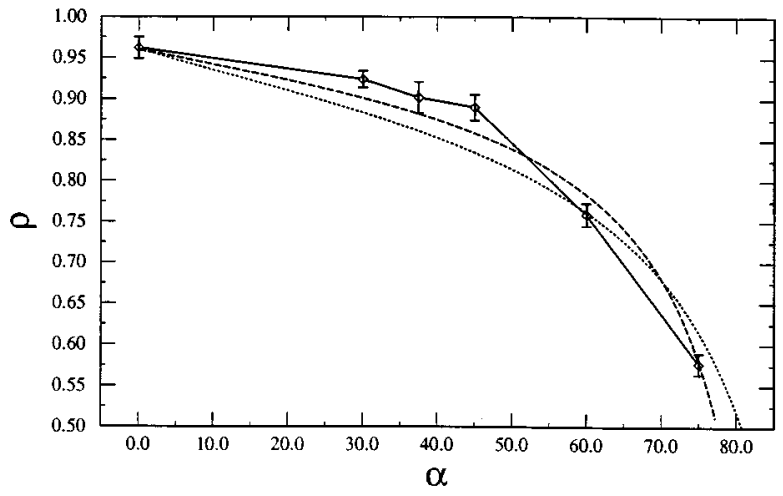

(b)

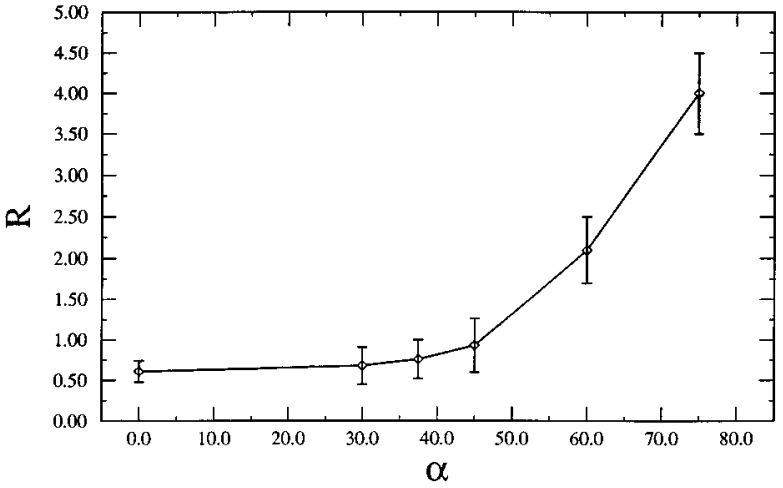

FIG. 8. Deposition angle dependence of the (a) film density $\rho$ and (b) mean surface roughness $R$, for films deposited at $T=0.125 \epsilon / k$ and $E_{b}=0.80 \epsilon$. The simulation data is represented by the symbols and solid line, the dashed curve is the prediction of Eq. (4a), and the dotted curve is obtained from Eq. (4b). The surface roughness data reflects an average of five measurements per simulation (spaced equally in time) for three independent simulations per deposition angle, except for $\alpha=60^{\circ}$ and $75^{\circ}$ where the surface roughness data were only obtained at the end of each of three simulations.

the columns cross from one side of the sample to the other. It is reasonable to expect that when the column spans the entire system width, the microstructure may be influenced by the system size: in particular, for large $\beta$, the finite system width will impose an artificially small column separation. Therefore, we performed a series of simulations for deposition angles of $\alpha=45^{\circ}, 60^{\circ}$, and $75^{\circ}$ on substrates of varying width from 40 to $120 a_{0}$. The dependence of the microstructure on substrate size is shown in Fig. 10 for $\alpha=60^{\circ}$. While the void density does not change significantly with substrate width, there is a significant increase in $\beta$ with increasing column width. Figure 11 shows the variation of $\beta$ with system width for $\alpha=45^{\circ}, 60^{\circ}$ and $75^{\circ}$. Despite the relatively poor statistics (three simulations for each data point) in this figure, it is clear that $\beta$ increases with substrate width for $\alpha=60^{\circ}$ and $75^{\circ}$ and then saturates at large substrate width. Comparison of these large $\alpha$ simulation results with those obtained at $\alpha=45^{\circ}$ shows that the width at which $\beta$ saturates increases with increasing $\alpha$. For $\alpha=45^{\circ}$, Fig. 11 shows that $\beta$ has saturated at widths as small as $40 a_{0}$. Therefore, based upon these results and the desire to minimize the computational
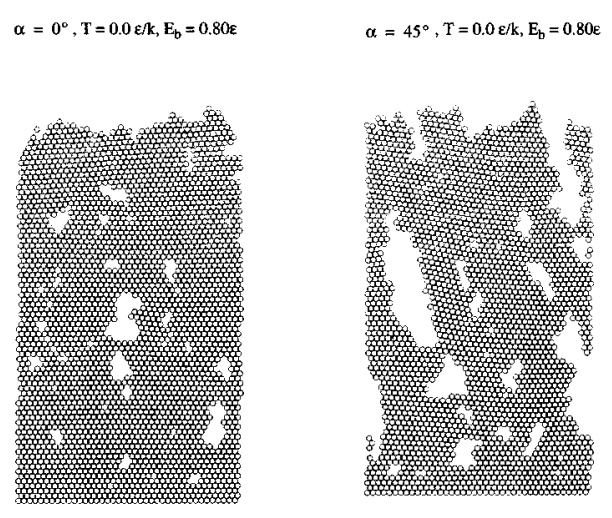

(b)

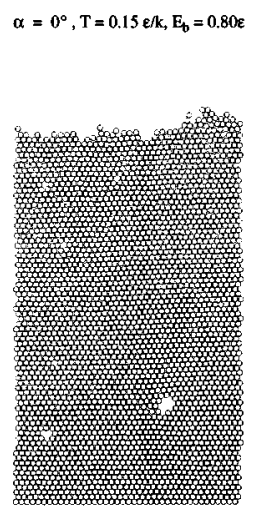

FIG. 9. Comparison between typical film microstructures grown at normal $\left(\alpha=0^{\circ}\right)$ and oblique $\left(\alpha=45^{\circ}\right)$ atom incidence.

burden associated with these simulations, we chose to perform all of the simulations with a substrate width of $40 a_{0}$, except for those at $\alpha=60^{\circ}$ and $75^{\circ}$, for which $\beta$ does not saturate until the substrate width is of order $100 a_{0}$.

Paik et al. ${ }^{6}$ performed a series of MD simulations of film growth as a function of deposition angle. In these simulations, they seeded individual columns by putting local bumps on the substrate prior to deposition. They did this because they argued that without these seeds, columnar structures would not form. This is not consistent with the present simulation results that show that there is a clear relationship between the columnar structure/void tracks and the surface roughness (cf. Fig. 1 in Ref. 6 with Fig. 10 here). Since the roughness forms naturally after sufficient film growth, we believe that the need of Paik et al. to seed the columns was based upon not depositing a sufficiently thick film for the temperature, deposition kinetic energy, and deposition angle employed. We note that as the temperature or deposition kinetic energy increases, it becomes necessary to grow to considerably greater thicknesses than at low $T$ and $E_{b}$ in order to observe the void tracks. Similarly, increasing deposition angles decreases the thickness of the film necessary to see columnar structures form since the surface roughness of large $\alpha$ films develops quicker and is larger than for low $\alpha$ films. 

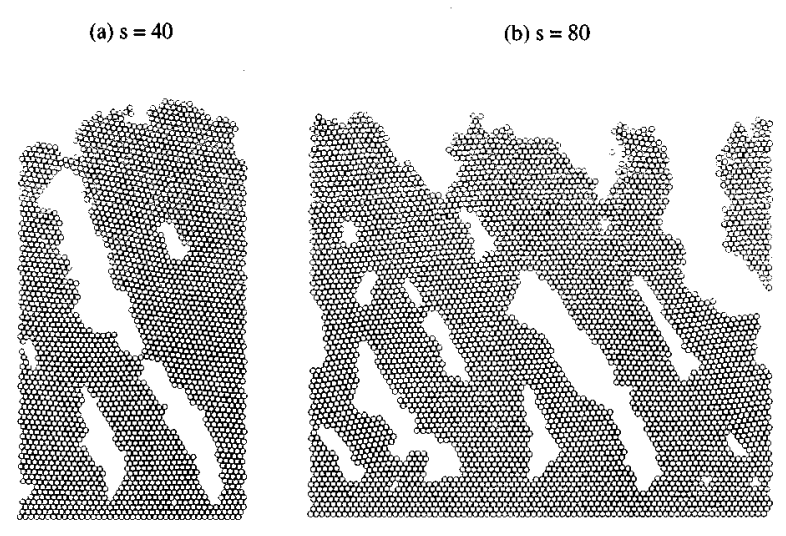

(c) $\mathrm{s}=120$

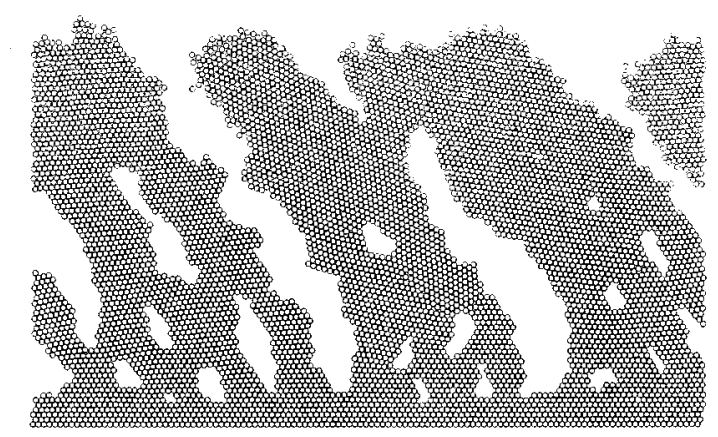

FIG. 10. Typical microstructures for films grown on substrates with different widths at $T=0.125 \epsilon / k, \alpha=60^{\circ}$, and $E_{b}=0.80 \epsilon$.

Column angles, void tracks angles, and elongated void orientations are all the same in the present simulations (in cases where they can all be observed). This relationship is attributable to the fact that they all can be traced to the angles of the deep surface depressions (voids that have not yet pinched off) that form during film growth. The angles at which these depressions occur can be traced back to shadowing, as described by Dirks and Leamy. ${ }^{4}$ The average column/void track/void angles are tabulated in Table I as a function of deposition angle at fixed temperature and depo-

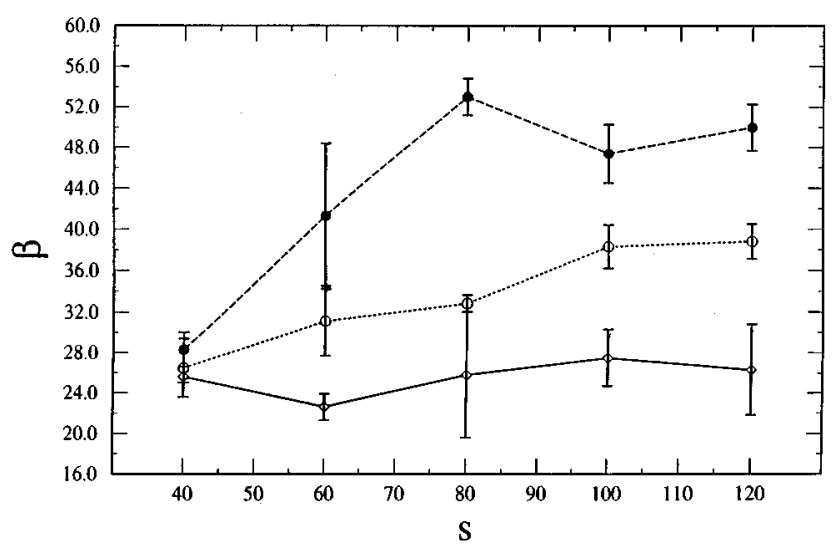

FIG. 11. Dependence of the column angle $\beta$ on substrate size $s$ for films grown at $T=0.125 \epsilon / k, E_{b}=0.80 \epsilon$ and $\alpha=45^{\circ}$ (solid curve), $60^{\circ}$ (dotted curve) and $75^{\circ}$ (dashed curve).
TABLE I. Void track angles $\beta$ as a function of deposition angles for simulations performed at $E_{b}=0.80 \epsilon$ /atom and $T=0.125 \epsilon / k_{B} \cdot \beta, \beta_{1}$ and $\beta_{2}$ correspond to the simulation data [Eq. (2)] and the tangent law [Eq. (1)], respectively.

\begin{tabular}{rcrc}
\hline$\alpha$ & $\beta$ & $\beta_{1}$ & $\beta_{2}$ \\
\hline 0.0 & $\ldots$ & 0.0 & 0.0 \\
30.0 & $\ldots$ & 26.1 & 16.1 \\
37.5 & $\ldots$ & 31.6 & 21.0 \\
45.0 & $25.6 \pm 0.6$ & 36.6 & 26.6 \\
60.0 & $38.8 \pm 1.7$ & 45.5 & 40.9 \\
75.0 & $50.0 \pm 2.3$ & 53.2 & 61.8 \\
\hline \hline
\end{tabular}

sition kinetic energy. In addition, Table I also shows the predictions of the well-known tangent law [Eq. (1)] and the relationship between deposition angle and column angle predicted by Tait et al. $^{7}$ based upon a geometric model [Eq. (2)]. Since no well-defined void track/column angle could be determined at $\alpha=0^{\circ}, 30^{\circ}$, and $37.5^{\circ}$ at the temperature and deposition kinetic energy employed in these simulations, these data were omitted from the table. (We chose not to seed columns, as Paik et al. ${ }^{6}$ did, since this arbitrarily fixes the column spacing.) Comparison of the data in Table I with Eqs. (1) and (2) suggest that both the tangent law [Eq. (1)] and Eq. (2) provide reasonable fits to the simulation data. However, it appears that the tangent law [Eq. (1)] works somewhat better for low $\alpha$ (i.e., $\alpha \leqslant 60^{\circ}$ ), while Eq. (2) is better at large $\alpha$. The good agreement between the simulations and the tangent law for $\alpha \leqslant 60^{\circ}$ is similar to the type of agreement found between the tangent law and experimental data. ${ }^{3,4}$ Further, at large $\alpha$ where there is noticeable disagreement between the simulation and the tangent law, we find that the experimental data (see Fig. 2 in Ref. 4) deviates from the tangent law in the same direction.

The general features of the variations in the microstructure with varying deposition angle were explained in terms of shadowing effects (see above). Increasing deposition angle increases column angle $(\beta)$ and surface roughness, and decreases the density. Further, as the deposition angle and column angle increase, the material changes from one in which the film is more or less continuous at all heights (i.e., isolated voids) to one in which the columns are well defined and the sizes of the voids are comparable to the column sizes (cf. $\alpha=30^{\circ}$ and $\alpha=75^{\circ}$ in Fig. 7). This explains why the density decreases with increasing $\alpha$. This correlation between column angle and void width was noted earlier by Dirks and Leamy ${ }^{4}$ and by Paik et al., ${ }^{6}$ who predicted the relationship between film density and deposition angle based upon the tangent law. Dirks and Leamy ${ }^{4}$ suggested that

$$
\rho(\alpha)=\rho(0)[1-A \tan (\alpha)],
$$

and Paik et al. ${ }^{6}$ suggested that

$$
\rho(\alpha)=\frac{\rho(0)}{1+B \tan (\alpha)},
$$

where $A$ and $B$ are constants. We compare these two relationships with our simulation data in Fig. 8, where the parameters $A$ and $B$ were chosen to yield the best fit with simulation data. Both Eqs. (4a) and (4b) yield reasonably 
good agreement with the simulation data. However, we observe that the agreement is somewhat better for Eq. (4a) than for Eq. (4b). The value of $A$ determined by fitting to Eq. (4a) is $0.106 \pm 0.003$, and the value of $B$ determined by fitting to Eq. (4b) is $0.150 \pm 0.006$. The value of $A$ determined by fitting to two experiments on amorphous Ge is approximately $0.075 ;^{3-5}$ which is within approximately $30 \%$ of the simulation value. These results show that these simulations are in reasonable agreement with experiment and that Eq. (4a) is a good description of the effect of deposition angle on density. The slight variation in the value of $\alpha$ between simulation and experiment is likely due to the fact that the simulations were performed in two dimensions, while the experiments were inherently three dimensional.

\section{CONCLUSIONS}

Atomistic, molecular dynamics simulations were employed to investigate the relationship between film microstructure and deposition conditions; namely, substrate temperature, deposition kinetic energy and deposition angle. By enhancing atomic mobility, substrate temperature and deposition kinetic energy were shown to play similar roles in modifying the microstructure: increasing temperature and deposition kinetic energy leads to fewer voids, smaller voids, smoother surfaces and higher film density. As the deposition angle increases, the film microstructure changed from a dense film, with few voids, to a microstructure in which nearly colinear tracks of elongated voids form and, finally, to a highly porous structure of well-formed columns. The angle along which the voids were elongated and the orientation of the void tracks were found to be the same and this angle $\beta$ increases with increasing deposition angle. The angle at which the columns were oriented followed the same trend with deposition angle as does $\beta$. Void formation, void alignment into tracks and the columnar structure are all attributable to shadowing effects, which become more pronounced with increasing deposition angle. Simulations were per- formed for a series of substrate width and the column/void track angles were shown to saturate at large, deposition angle-dependent widths. The variation of the column/void track angle $\beta$ with deposition angle $\alpha$ was found to fit the classical tangent law for low angles, but were overpredicted by the tangent law at large angle $\alpha>60^{\circ}$. This result is consistent with experimental observations in amorphous Ge. The simulations showed that the column angle $\beta$ decreased slowly with increasing deposition kinetic energy due to increased surface mobility.

\section{ACKNOWLEDGMENTS}

This research was supported by the U.S. Advanced Research Projects Agency and the U.S. Air Force Office of Scientific Research through MURI Grant No. F49620-95-10524.

${ }^{1}$ A. Movchan and A. V. Demchishin, Phys. Met. Metallogr. (U.S.S.R) 28, 83 (1969).

${ }^{2}$ J. M. Nieuwenhuizen and H. B. Haanstra, Philips Tech. Rev. 2787 (1966).

${ }^{3}$ N. G. Nakhodkin and A. I. Shaldervan, Thin Solid Films 10, 109 (1972).

${ }^{4}$ A. G. Dirks and H. J. Leamy, Thin Solid Films 47, 219 (1977).

${ }^{5}$ D. K. Pandya, A. C. Rastogi, and K. L. Chopra, J. Appl. Phys. 46, 2966 (1975).

${ }^{6}$ S. M. Paik, S. Kim, I. K. Schuller, and R. Ramirez, Phys. Rev. B 43, 1843 (1991).

${ }^{7}$ R. N. Tait, T. Smy, and M. J. Brett, Thin Solid Films 226, 196 (1993).

${ }^{8}$ K.-H. Müller, J. Appl. Phys. 58, 2573 (1985).

${ }^{9}$ S. Müller-Pfeiffer, H. van Kranenburg, and J. C. Lodder, Thin Solid Films 213, 143 (1992)

${ }^{10}$ P. Meakin and J. Krug, Phys. Rev. A 46, 3390 (1992).

${ }^{11}$ P. Smilauer, M. R. Wilby, and D. D. Vvedensky, Phys. Rev. B 47, 4119 (1993).

${ }^{12}$ P. Smilauer, M. R. Wilby, and D. D. Vvedensky, Phys. Rev. B 48, 4968 (1993).

${ }^{13}$ R. W. Smith and D. J. Srolovitz, J. Appl. Phys. 79, 1448 (1996).

${ }^{14}$ F. F. Abraham, in Melting, Localization, and Chaos (Elsevier, New York, 1982), p. 75.

${ }^{15}$ S. Toxvaerd, J. Chem. Phys. 69, 4750 (1978).

${ }^{16}$ W. W. Mullins, J. Appl. Phys. 30, 77 (1959). 